\title{
Interferometric Measurement and Numerical Comparisons of Supersonic Heat Transfer Flows in Microchannel
}

\author{
Yuya Takahashi ${ }^{a}$, Lin Chen ${ }^{\text {b,c, *, Junnosuke Okajima }}{ }^{b}$, Yuka Iga ${ }^{b}$, Atsuki Komiya ${ }^{b}$ and Shigenao \\ Maruyamab \\ ${ }^{a}$ Graduate School of Engineering, Tohoku University, Aoba 6-6, Aramaki-aza, Aoba-ku, Sendai, \\ 980-8579 Japan \\ ${ }^{b}$ Institute of Fluid Science, Tohoku University, Katahira 2-1-1, Aoba-ku, Sendai 980-8577, Japan \\ ${ }^{c}$ Overseas Research Fellow of Japan Society for the Promotion of Science (JSPS), Japan
}

\begin{abstract}
With the fast development of electronic systems and the ever-increasing demand of thermally "smart" design in space and aeronautic engineering, the heat transfer innovations and high heat flux challenges have become a hot topic for decades. This study is aimed at the effective cooling heat transfer design by super-/sub-sonic air flow in microscale channels for high heat flux devices. The design is based on the low temperature flows with supersonic expansion in microscale, which yields a compact and simple design. By careful microelectromechanical process, microscale straight and bumped channels (with simple arc curve) are fabricated and experimentally tested in this study. The microscale flow field and density distributions under new designs are visualized quantitatively by an advanced phase-shifting interferometer system, which results are then compared carefully with numerical simulations. In this study, large differences between the two designs in density distribution and temperature changes (around $50 \mathrm{~K}$ ) are found. The high heat flux potential for supersonic microchannel flows is realized and discussion into detail. It is confirmed that the bump design contributes significantly to the heat transfer enhancement, which shows potential for future application in novel system designs.
\end{abstract}

Keywords: Interferometer, Heat transfer, Supersonic flow, Microchannel, Numerical Simulation

\footnotetext{
* Corresponding author. Tel.: 81-22-217-5269 Fax: 81-22-217-5244

Email: chenlinpkucoe@gmail.com; chenlintohoku@pixy.ifs.tohoku.ac.jp

$1 / 29$ 


\section{Introduction}

Microchannel fluid dynamics and heat transfer problems have become one of the hot topics in engineering fields, due to the wide application of microscale devices and the ever-increasing heating/cooling demands [1-3]. It is reported in recent years that the power density of new generation integrated circuits has increased to higher than $1.0 \mathrm{MW} / \mathrm{m}^{2}$ [1]. The fast heating/cooling and thermal control process in aeronautics have also put forward new challenges, which require both miniaturized (and compact) design and high heat flux capacities [4-5]. In order to solve that problem, many researchers have been focused on the microfluidics related analysis, such as the thermal resistance examination, superconducting, heat transfer fluids, novel designs in microscales [5-6], etc.

However, it is still difficult to meet the high standard demand, as the microscale flow and heat transfer potentials are yet to be improved. For the enhancement of heat transfer behaviors, and mainly for cooling performance, large numbers of studies have been published in open literature. The focus on microchannel flows is one major proposal due to its relatively higher performance and the easy application for mini- and micro- sized devices. For single-phase flows, several representative studies [4-9] have experimentally and partial-numerically revealed the basic performance of circular and rectangular microchannels and their integration for cooling devices. Also, many working fluids, such as gas flow of incompressible and compressible designs, microchannel geometry modifications, as well as nano-fluid flow systems, have been conducted to test the microscale heat transfer performances [10-14]. In the study of Wang and his colleagues [10, 14], the microchannel through flows with top-truncated double-layers are numerically discussed, and an inverse method are further developed for optimization later. Their design showed generally $24 \%-40 \%$ reduction of thermal resistance in the numerical simulations, which is promising for future real application development. However, the main designs are still largely dependent on the pumping power range for those studies. It is found that high pressure or multi-phase flows generally yield higher performance, but it still diverges from case to case (and depend on specific designs) [15-18]. Recently, in a novel microscale heat transfer design with supercritical fluids [19-21] has also been conducted and it indicates that the fluid choice and channel design/control are both important factors for improving heat transfer performance. From those existing studies, limitations are seen for both forced kind of heat transfer design and natural convection kinds. If the target is to achieve high heat flux around $1.0 \mathrm{MW} / \mathrm{m}^{2}$, much higher refrigerant flow rate or phase change rate are necessary [1-3, 22]. Also the microscale 
fabrication and detailed system design and thermal efficiency analysis still remain as problems yet to be solved [23-24]. New proposals for the microscale fluidic designs to improve the heat transfer and flow control are still needed in this field.

The current study is focused on a new design of utilizing supersonic flow into microscale heat transfer apparatus. The supersonic flow can be generated by adiabatic expansion, and it generates shock wave and lower temperature downstream flow condition in the microscale [25-28]. In this study, a newly proposed microchannel with simple bump sections, as a convergent-divergent section, is experimentally fabricated and tested as a candidate for the high heat flux cooling device. By generating supersonic flow, low-temperature downstream region can be realized for possible heat transfer section. Fundamental investigations of supersonic fluid flow realization and microchannel design have been made in previous trials [29-30]. However, only the general heat transfer boundary or heat transfer effects on the flow (i.e., the flow dynamics) have been discussed in literature. To utilize the low temperature flow potential, the basic supersonic flow in microscale can be proposed in this study for heat transfer goals.

And in this study, it is set as a goal to visualize the detailed density behaviors and temperature evolutions in the newly designed supersonic microchannel. he temperature distribution is important to evaluate the heat transfer performance between the flow and walls, i.e., cooling performance of the heat sink in microscale. However, it is seen as a very difficult task to visualize the flow and density/temperature field inside microchannel. In literature, very few experimental studies of the microchannel flow visualization of the flow and density field can be found [30-31]. In this study, for a feasibility comparison, a newly designed phase-shifting Mach-Zehnder interferometer system is also utilized as a non-intrusive and quantitative measurement technique [31-32]. Generally, it is difficult to measure the flow inside the microchannel as the optical length difference and refractive index measurement is inadequate for well data manipulation. The interferometer system is proposed in this study to visualize the basic density changes inside the microchannel to test the supersonic flow heat transfer and compare with the numerical simulation results.

The overall objective of this study is to confirm the availability of the bumped channel for the micro heat sink, and to measure the density distributions of supersonic flow inside the microchannel using a phase-sifting interferometer. The following sections will first show the design, the fabrication of the new heat transfer channel, and then a newly proposed interferometric measurement system is 
introduced. After that the experimental results are compared with numerical simulation data for basic density field and heat transfer analysis. Other details of heat transfer characteristics, comparisons between straight channel and newly designed bump channels, and the effects of supersonic flow designs are also discussed into detail in this study.

\section{Microchannel Design and Interferometer Measurement System}

\subsection{Microchannel design and fabrication}

In order to evaluate the effect of the micro-nozzle on the density distributions, two types of the microchannel were designed. One has a straight channel shown in Fig. 1(a). The other one has a micro-nozzle at the inlet region of the channel as shown in Fig. 1(b), which was named as bumped nozzle. The shape of the bumped nozzle follows a simple arc curve that was easy for fabrication. The advantages of bumped nozzle were also discussed in previous studies [29-30]. Silicon plate was sandwiched between two TEMPAX $\odot$ plates by anodic bonding for visualization in this design. The main target of the current design is to test and compare the heat transfer flow between the newly designed bump channel and conventional straight channels. The detailed size and chamber flow system can also be found in Fig. 1 (a) and (b). For the real experimental system, the HITACHI BEBICON compressor (5.5U-9.5GB5/6) is used. For temperature measurement, T-type thermocouples are used. An automatic data logger (KEYENCE GR-3000) is used to record and transport data from the experiment.

The procedures for the fabrication of the proposed microchannels are shown in Fig. 2. The microchannels were fabricated on Si plate, which crystal orientation is (100) by the Deep Reactive Ion Etching (Deep-RIE) process. The entire size of this Si plate was $20 \mathrm{~mm}$ in width and depth and $500 \mu \mathrm{m}$ in thickness. The detailed size and bump design are fabricated according to the designs in Fig. 1. The length, width, and depth of the channel are 6000, 235.24, and $500 \mu \mathrm{m}$, respectively. In order to avoid the effect of the inlet/outlet region of the device, large reservoirs were placed at the inlet and outlet of the microchannel as also shown in Fig. 1.

\subsection{Interferometer measurement system and data process}

2.2.1 Principals and design of phase-shifting interferometer

In the current study, interferometry technique is used to measure the density field inside the 
microchannel. An interferometer basically use the interference patterns from the sample beam and the reference beam, with which the field information of the test sample can be measured and recorded in the form of pattern by a CCD camera. For better visualization of the detailed density distribution, a phase-shifting interferometer is utilized in this study. The phase-shifting technique is an image processing technique, which calculate and conflates brightness of the interferograms at different polarization angles [31-33]. Then, all pixel of a CCD camera detects phase difference data using the phase-shifting interferometer. Therefore, the refractive index can be obtained as the brightness of 256 steps in phase-shifted data of 8 bit gray scale. This is in contrast to the conventional interferometer, which can only obtain values in the brightest and darkest points on the fringe. A schematic phase-shifting system can be seen in Fig. 3.

In this study, three interferometers at different polarization angles $(0,2 / 3 \pi, 4 / 3 \pi)$ were used. This polarization angle was changed by a rotating polarizer. Thus, the phase-shifted data was calculated every $1 / 10 \mathrm{~s}$ by synchronism with a frame rate of the CCD camera. As a light source, a He-Ne laser of $632.8 \mathrm{~nm}$ in wave length was used. The estimated fringe number, $N$, and the number of data points calculated from the Gladstone-Dale relation are compared in Table 1, for traditional interferometer and the developed phase-shifting interferometer.

The Gladstone-Dale relation is shown in following equations [31-33].

$$
\begin{aligned}
& n=1+\rho \cdot K=1+\frac{p \cdot K}{R \cdot T} \\
& N=\Delta l(x, y) /\left(\frac{\lambda}{2}\right)=\frac{2}{\lambda} \int_{-\infty}^{+\infty}\left[n_{\mathrm{o}}-n_{\mathrm{r}}\right] \mathrm{d} z
\end{aligned}
$$

where, $n[-], n_{\mathrm{o}}[-], n_{\mathrm{r}}[-], \rho\left[\mathrm{kg} / \mathrm{m}^{3}\right], p[\mathrm{~Pa}], R[\mathrm{~J} /(\mathrm{kg} \cdot \mathrm{K})], T[\mathrm{~K}], \lambda[\mathrm{m}], K\left[\mathrm{~m}^{3} / \mathrm{kg}\right]$, and $\Delta l[\mathrm{~m}]$ denote refractive index, refractive index of test beam, refractive index of reference beam, density, pressure, gas constant, temperature, wave length of the light source, Gladstone-Dale constant, and difference of the optical path length, respectively. The optical path length is equal to the channel depth in the experiment. For the density evaluation, air is selected as the working fluid and the initial temperature is set at $300 \mathrm{~K}$.

As shown in Table 1, the expected fringe number is considerably small because of the short optical path length of the microchannel. The difference of optical path length, which is referred from the microchannel, is equal to the depth of the microchannel in this study. According to previous studies [29-30], the range of the density difference of the supersonic flow inside a microchannel was 
estimated to be between $1.0 \mathrm{~kg} / \mathrm{m}^{3}$ and $5.0 \mathrm{~kg} / \mathrm{m}^{3}$. It can be seen in Table 1 that even $2000 \mu \mathrm{m}$ of channel depth is not enough for the measurement using conventional interferometer. The phase-shift interferometer use in this study is able to produce the values of the phase difference at a phase resolution of $\lambda / 256$ and the phase resolution can be 100 times higher than with a conventional interferogram [31-33]. The using of current phase-shifting interferometer provided a method to capture the density field inside the microchannels. In addition, it should be noted that the depth of the microchannel should also be designed on the trade-off between the measurement needs and the fabrication technology.

\subsubsection{Experimental measurement system and data process}

The schematic diagram of the phase-shifting interferometer measurement system is shown in Fig. 3. The phase-shifted data will be obtained by the post-processing of the interferogram, which is measured by the transient system together with the microfluidics flow control. These experiments were conducted under the condition of controlled temperature of $300 \mathrm{~K}$ (while the room temperature is initially at $298 \mathrm{~K}$; which will not affect the experiment as the system is well insulated during the tests). Temperatures are also measured at the inlet and outlet of the two fluid chambers for the reference temperature values of the system calculation. As air is used for the experimental test, the procedure is very simple: (1) firstly the microchannel flow system is set up and stabilized on the optical stage; (2) air flow tests are conducted to clear the flow passage and test tightness of the system; (3) calibration of interferometer system to obtain stable background interferogram; (4) start the data accumulation system; (5) start the compressor and flow valves to generate microchannel flow for the designed conditions; (6) data accumulation; (7) when finished, turn off the optical system and flow system. During the experiment, the compressed air flow passes through the designed microchannels from inlet to the outlet reservoirs, where the pressure at the inlet and outlet were designed as $0.7 \mathrm{MPa}$ and $0.1 \mathrm{MPa}$, respectively.

The data accumulation was performed less than $10 \mathrm{~s}$, and during which the TEMPAX ${ }^{\odot}$ glasses were cooled by the expanding flow. The temperature decrease of the glass causes the phase difference of the laser beam due to the temperature dependence of the refractive index, which is then compared with numerical results. In the current experiment, the interferograms are detected by CCD camera and then converted to the refractive index and density distributions based on Gladstone-Dale relation (Eq. (1)-(2)). 


\subsubsection{Measurement uncertainty analysis}

The measurement uncertainty analysis of experimental data should be done to ensure the present data. The uncertainty of the measurement may be affected by the nonlinearity of the CCD sensor and optical devices, as well as the errors in data processing procedures. The temperature and measurement errors are less than $\pm 0.1^{\circ} \mathrm{C}$ and $\pm 0.075 \%$ of the measure value. In this experimental set-up, the main factors of bias errors are location measurement error and intensity measurement error. The resolution of measured location was approximately $0.01 \mathrm{~mm}$, which corresponds to the real length in one pixel of the bitmap image. The CCD camera detects the density distribution with precise position (i.e. less than 1pixel measurement error). Therefore, the bias error due to the measurement error of location could be estimated as a value of $\pm 0.03 \%$, which can be negligible. Concerning the measurement error of intensity, the resolution of detected intensity datum is $0.01 \mathrm{~kg} / \mathrm{m}^{3}$, which corresponds to $\pm 0.27 \%$. More details of theoretical analysis of uncertainties in phase-shift interferometers can be found in our previous reports on interferometer designs [31-33].

\section{Numerical Formulations}

\subsection{Physical and numerical model}

Numerical simulations are performed using ANSYS ${ }^{\circledR}$ FLUENT 13 software to compare with the measurement data. The flow was assumed as a steady state and laminar for simplicity of discussion. In this calculation, the temperature dependence of the thermo-physical properties was taken in account during the modeling and iteration. The width and length of the channel are set to be similar with the experimental design as shown in Fig. 1. A clear numerical calculation domain set-up with basic geometric and design parameters are shown in Fig. 4. In the design, horizontal through flow microchannel and bumped channel settings are discussed and compared for feasibility analysis of new proposals in microscale heat sinks.

\subsection{Numerical Parameters and Procedures}

Classical Navier-Stokes equations are used to solve the current problem, together with the energy equation and the equation of state written for compressible fluid:

- mass conservation equation 


$$
\frac{\partial \rho}{\partial t}+\nabla \cdot(\rho \vec{v})=0
$$

- Navier-Stokes equation

$$
\frac{\partial(\rho \vec{v})}{\partial t}+\nabla \cdot(\rho \vec{v} \vec{v})=\nabla \cdot\left[\mu\left(\nabla \vec{v}+(\nabla \vec{v})^{\mathrm{T}}\right)-\frac{2}{3} \nabla \cdot \vec{v} I\right]-\nabla p
$$

- energy equation

$$
\begin{aligned}
& \frac{\partial(\rho E)}{\partial t}+\nabla \cdot\{\vec{v}(\rho E+p) \cdot\}=\nabla \cdot\left\{k_{\text {eff }} \nabla T+\left(\overline{\bar{\tau}}_{\text {eff }} \cdot \vec{v}\right)\right\} \\
& E=h_{e}-\frac{p}{\rho}+\frac{\vec{v}^{2}}{2}
\end{aligned}
$$

Where $\bar{\tau}_{\text {eff }}, \mu, h_{e}, I$, and $k_{\text {eff }}$ represent stress tensor, viscosity coefficient, enthalpy, unit tensor and effective thermal conductivity, respectively. It should be noted that the fluid is treated compressible and especially the relationship in the equation of state are carefully coupled with the energy equation and Navier-Stokes equation for each iteration step. Also, the channel is seen as smooth one and the horizontal wall conduction is neglected for basic model study.

The current model uses air as the working fluid. And as the focus of the current paper is on the channel design, idea gas model is used for first step and basic feasibility analysis. For the transient stability evolution analysis, a finite volume solution method was used, and a numerical model was developed using ANSYS FLUENT and validated in previous studies [29-30]. The Roe's Flux Difference Splitting (Roe-FDS) method is used for the flux estimation in the current model. The momentum terms and energy terms in the governing equations are iterated with a second-order upwind scheme, which uses the upstream values and gradients to compute the control volume face values. For the near-wall simulation, a non-slip boundary condition is used. Geometric and boundary conditions can be found in Fig. 4. The pressure at the inlet and outlet were 0.7 and $0.1 \mathrm{MPa}$, respectively. Total temperature was set to $300 \mathrm{~K}$ at the inlet, which is the same as the experimental tests.

In the current numerical study, the iteration time step of the numerical iteration ranges between $10^{-4}$ and $10^{-2}$ to capture the details of the boundary effects. The convergence limitation is $10^{-3}$ for the residuals of continuity and velocity and $10^{-6}$ for the energy term in each step of iteration. Other numerical details can be found in previous studies [29-30]. For the first step, the microchannel length is set as $L=6.0 \mathrm{~mm}$ with channel height around $200 \mu \mathrm{m}$ to $300 \mu \mathrm{m}$ (with the calculated Knudson number smaller than $\left.1.0 \times 10^{3}\right)$. Non-uniform grids are used in the calculation domain with mesh grids 
ranging from $1.0 \times 10^{5}$ to $2.0 \times 10^{6}$ for different case configurations and are refined specifically for the boundary and internal bump areas during the initial studies to ensure that mesh-independent results are obtained. The current numerical methods have also been tested and verified in previous studies with microscale channel supersonic flows [29-30].

\section{Results and Discussion}

\subsection{Interferometric measurement results}

\subsubsection{Straight channel results and comparison}

The typical phase-shifted data and measured density distribution of the straight channel are shown in Fig. 5. The density distribution on the microchannel centerline is shown in Fig. 5(a), which is developed by using Gladstone-Dale relation. The spatial-averaged values are used for the evaluation and comparison of the density distribution. Here, the 5 pixels around the centerline of the channel were used for the spatial average and the data for three seconds are used for the time-average calculations. As shown in Fig. 5(a), the values of density varied due to the pressure drop inside the microchannel, and its distribution can be provided through phase-shifted data. The density distribution exhibited a rapid decrease in the horizontal direction due to the supersonic flow development at the inlet of the channel. The free jet stream was observed at the end of the channel. As such, the flow density and temperature is decreased sharply under rapid adiabatic expansion. Also it can be seen from Fig. 5 that, the current new phase-shifting interferometer system could measure the density distributions at all pixels. In addition, the brightness distribution could be detected in the flow field even if the fringe number is very small, which is advantageous over conventional interferometers.

\subsubsection{Bumped Channel Results and Comparison}

The phase-shifted data and measured density distribution of the bumped channel are shown in Fig. 6 . The density distributions were evaluated on/around the centerline. Average values were used same as the case of straight channel. As shown in Fig. 6, the density distributions were approximately the same with the straight channel, except the decrease at the inlet region. At the bumped section, compared to the case of the straight channel, the values show larger decrease caused by the supersonic expansion due to the convergent-divergent shape and the appropriate ratio of the cross-section. 


\subsection{Experimental Performance and Numerical Comparisons}

\subsubsection{Density field comparison between experimental and numerical simulations}

The measured density around the center line of the straight channel and the bumped channel as a function of the position are shown in Fig. 7 with the calculated density distributions on the center line of the channels. It should be noted that the simulated and measured flow rate for the designed microchannel are $8.47 \times 10^{3} \mathrm{~cm}^{3} / \mathrm{min}$ and $8.58 \times 10^{3} \mathrm{~cm}^{3} / \mathrm{min}$, which indicates good control of the flow system. And as shown in Fig. 7, the measured density distribution inside a straight channel shows good agreement with that of the numerical simulation. For the bumped channel, the range and the trend of density were also approximately the same as the case of straight channel, except for the inlet region of the channel.

The calculated and the measured density distributions of the bumped channel show obvious decrease in the inlet region due to the existence of the bump. After the throat, the measured density distribution was once increased by the pressure loss inside the channel and then gradually decreased. Near the outlet region, for example the position of $x=5 \mathrm{~mm}$, the density values decreases rapidly due to the flow expansion. Such quick decrease shows good agreement between the measurement and the numerical simulation for both channels, thus to conclude that the well-established interferometer system is capable to capture the density field inside microchannels under supersonic flow conditions.

On the other hand, the calculated density distribution of the Bump channel was gradually increased toward the channel end. Differences between numerical curves and experimental results are found for 5 $\mathrm{mm}<\mathrm{x}<8 \mathrm{~mm}$ in Fig 7. Though it does not affect the basic trend, but it reveals several important factors to be considered in microchannel flows: at first, these numerical simulations were conducted as two dimensional and adiabatic wall conditions for simplicity of first step analysis. In the measurement, the wall conditions of the microchannel device had kind of an isothermal condition of the controlled temperature of $300 \mathrm{~K}$. Thus, the three dimensional effect and the heat transfer from the channel wall were ignored in the numerical simulations. Second, when the density was evaluated in measurement data, the temperature dependency of Gladstone-Dale constant was ignored and that temperature was assumed as controlled experimental temperature. Third, the accuracy of the channel shape and roughness of the channel wall, which were caused by the MEMS fabrication processes. The thickness of the Si plates, which was equal to the channel depth, is large for the etching process. Thus, the shape and the size differences were occurred despite of the crystal orientation was selected to make parallel walls, 
especially in the case of the bumped channel. In order to against these problems, temperature effect on the density distribution and the fabrication process will be considered in the future.

\subsubsection{Flow behaviors of straight channel design}

The calculated Mach number, density, and temperature distributions of the straight channel are shown in Fig. 8. As shown in Fig. 8(a), pressurized air flow is simulated under a through-pressure difference of 0.6 MPa and gradually accelerated due to the supersonic flow expansion. The Mach number at the inlet is smaller than 1.0 , and it is gradually accelerated to higher than 1.0. When the maximum Mach number is reached, the flow temperature decreased to its minimum of $135 \mathrm{~K}$ due to the adiabatic expansion, as shown in Fig. 8(b). The maximum Mach number and the minimum temperature is about 1.44 and $250 \mathrm{~K}$ at the outlet region of the microchannel, respectively. The density distribution shows a similar evolution trend with the temperature distribution. The values were decreased with decreasing temperature and pressure. In addition, the flow field was similar to the measured phase-shifted data shown in Fig. 5(a).

\subsubsection{Flow behaviors of the bumped channel design}

The calculated Mach number, temperature, and density distributions are shown in Fig. 9(a)-(c) for bumped channel design. As shown in Fig. 9(a), the air flow was accelerated from the inlet by the pressure gradient and the flow expansion till the downstream region, which is similar to the situation of straight channel. However, the flow in bumped microchannel is more accelerated due to the supersonic expansion at the bumped throat. The Mach number at the inlet is smaller than 1.0. Then the flow is choked and the Mach number reaches 1.0 at the throat and then further increased to about 1.5. The Mach number distribution was more than 1.0 downward region inside the channel after the throat. The maximum Mach number of 2.72 is observed at the free jet stream. As shown in Fig. 9(b), the maximum Mach number of 1.48 and the minimum temperature of $209 \mathrm{~K}$ inside the channel were observed at the end of the bumped section. Thus, the temperature was decreased about $90 \mathrm{~K}$ on the center line of the channel due to the adiabatic expansion by the bump design. It should be noted that the center line value is only selected for the convenience of discussion, which data shows basic evolutions of parameters along with the mainstream flow direction inside the microchannel. Cross section distributions of parameters are not discussed due to the good homogeneity of cross area in microscale. The density 
distribution has the same tendency with the temperature distributions as described above. The values of density were decreased with decreasing temperature, the same as the case with straight channel.

\subsubsection{Characteristics of the bump design}

The detailed effects of bump design are analyzed by further comparison of measured data between the straight channel and new design. The measured density distribution of the two designs are plotted and compared in Fig. 7 under the same initial and boundary conditions. It can be seen from Fig. 7 that the inlet region with sharp drop of density are of similar trend for both designs. However, for the bumped design, the density shows large decrease due to supersonic expanding flow. It is seen from Fig. 9(a) that the Mach number reaches 1.0 at the throat. In addition, the cross-section ratio of the throat to the channel is set to 1.1762 , which is determined according to the Mach number of 1.50. After the bumped section, tendency of density distribution is approximately the same till the end of the channel. In this region, the flow is mainly controlled by the friction pressure drop in the microchannel. It should be noted that for real microchannel fabrication and testing, the effect of surface roughness inside the microchannels should be taken care of, as such effects will control the basic pressure losses of the flow. It is shown that the low temperature flow, which is generated at the throat region, can be maintained through the microchannel. For real applications, noise of supersonic flow and the flow control still need further development.

Further analysis of the measured results show that the density difference of the straight and the bumped channel is about $1 \mathrm{~kg} / \mathrm{m}^{3}$ near the downstream of the throat. And from the numerical simulations, that value is very close to the experimental one. Further, the temperature difference between the straight and the bumped channel, caused by the existence of the bump design, is estimated to be around $52.0 \mathrm{~K}$ with the pressure of $0.3 \mathrm{MPa}$ at the downstream of the throat, as shown in Fig. 10. For the evaluation of the cooling performance of the heat sink, which includes the supersonic microchannel for the high heat flux applications, the temperature distribution is imperative. Then it can be concluded that the low temperature flow by the bumped channel design in this study is feasible for enhancement of system performance. The heat flux on the wall of the microchannel can be evaluated using calculated temperature distribution when the density distributions are same in measurement and numerical simulations. It is also suggested that, based on the supersonic flow in microchannels, the detailed geometric design and cooling performance optimizations be conducted in the future. 


\section{Conclusion}

A newly designed microscale channel with bumped section has been experimentally tested and numerically compared in this study. The new design utilized the supersonic air flow inside microchannel so as to enhance the heat transfer performance. In order to test the feasibility of the new design, an advanced phase-shifting interferometer system is utilized for the measurement of microchannel flow density. The measured supersonic air flow data are then compared with numerical simulations for both straight channel design and bumped designs. The main findings are summarized as follows.

(1) By careful system design and orientation, the transient and dynamic density fields inside the microchannel with only $500 \mu \mathrm{m}$ in depth are successfully obtained for both the straight channel and bumped channel.

(2) The density fields were numerically simulated and compared with the experimentally results with good agreement. The basic trends meet well while some differences were observed due to channel roughness, three-dimensionality effect, etc.

(3) Comparison between the straight channel and the bumped channel show that the density of the bumped channel yields larger temperature decrease at the throat. It is confirmed that the low temperature supersonic air flow, which is required to improve the cooling performance of the supersonic microchannel, is successfully generated and maintained in the newly designed microchannel.

(4) The effect of the bump design is discussed and it is found that the temperature will be around 50.0 K lower than that of the straight channel for the new design, which indicates the potential for the new design for microscale heat transfer device. Further studies on the design and optimizations is recommended for future study.

\section{Acknowledgement}

This work is supported by JSPS (Japan Society for the Promotion of Science) Grant-in-Aid for

Challenging Exploratory Research (Mr. Y. Takahashi; Grant No. 52673530), JSPS Grant for Overseas Research Fellow (Dr. L. Chen; Award No. 16F16068), Institute for International Advanced Research 
and Education (Tohoku University), the Global COE program, "World Center of Education and Research for Trans-disciplinary Flow Dynamics" in Tohoku University, and also JST-CREST Grant (Program: Breakthrough on Multi-scale Interfacial Transport Phenomena in Oceanic Methane Hydrate Reservoir and Application to Large-Scale Methane Production).

\section{References}

[1] A. Danowitz, K. Kelley, J. Mao, J.P. Stevenson, M. Horowitz, CPU DB: Recording Microprocessor History, Queen - Processors 10 (4) (2012) 1-18.

[2] E. Pop, Energy Dissipation and Transport in Nanoscale Devices, Nano Res. 3(3) (2010) 147-169.

[3] R.J. McGlen, R. Jachuck, S. Lin, Integrated thermal management techniques for high power electronic devices, Appl. Therm. Eng.24 (2004) 1143-1156.

[4] P. Rosa, T.G. Karayiannis, M.W. Collins, Single-phase heat transfer in microchannels: The importance of scaling effects, Appl. Therm. Eng. 29 (2009) 3447-3468.

[5] X.F. Peng, B.X. Wang, G.P. Peterson, H.B. Ma, Experimental Investigation of Heat Transfer in Flat Plates with Rectangular Microchannels, Int. J. Heat Mass Trans. 38 (1995) 127-137.

[6] T.M. Adams, S.I. Abdel-Khalik, S.M. Jeter, and Z.H. Qureshi, An Experimental Investigation of Single-phase Forced Convection in Microchannels, Int. J. Heat Mass Trans. 41 (1998) 851-857.

[7] T.M. Harms, M.J. Kazmierczak, and F.M. Gerner, Developing Convective Heat Transfer in Deep Rectangular Microchannels, Int. J. Heat Fluid Flow 20 (1999) 149-157.

[8] G.M. Mala, and D. Li, Flow Characteristics of Water in Micro-tubes, Int. J. Heat Fluid Flow 20 (1999) 142-148.

[9] K. Kawano, M. Sekimura, K. Minakami, H. Iwasaki, and M. Ishizuka, Development of Micro Channel Heat Exchanging, JSME Int. J (B), Fluids Therm. Eng. 44 (2001) 592-598.

[10] C. Leng, X.D. Wang, T.H. Wang, An improved design of double-layered microchannel heat sink with truncated top channels, Appl. Therm. Eng. 79 (2015) 54-62.

[11] W.W. Liou, and Y. Fang, Heat Transfer in Microchannel Devices Using DSMC, J. MEMS. 10 (2001) 274-279.

[12] Y.P.T. Asako, S.E. Turner, and M. Faghri, Effect of Compressibility on Gaseous Flows in Microchannels, Int. J. Heat Mass Trans. 46 (2003) 3041-3050. 
[13] K. Hara, M. Inoue, and M. Furukawa, Heat Transfer in Mini-channel Gaseous Cooling, J. Environ. Eng. 2 (2007) 525-534.

[14] X.D. Wang, B. An, L. Lin, D.J. Leed, Inverse geometric optimization for geometry of nanofluid-cooled microchannel heat sink, Appl. Therm. Eng. 55 (2013) 87-94.

[15] M.B. Bowers, and I. Mudawar, High Flux Boiling in Low Flow Rate, Low Pressure Drop Mini-channel and Microchannel Heat Sinks, Int. J. Heat Mass Trans. 37 (1994) 321-332.

[16] R.K. Sarangi, A. Bhattacharya, R.S. Prasher, Numerical modelling of boiling heat transfer in microchannels, Appl. Therm. Eng. 29 (2009) 300-309.

[17] J.B. Marcinichen, J.R. Thome, and B. Michel, Cooling of Microprocessors with Micro-evaporation: A Novel Two-phase Cooling Cycle, Int. J. Refrig., 33 (2010) 1264-1276.

[18] J. Okajima, A. Komiya, S. Maruyama, Analysis of Evaporative Heat Transfer by Expansion Bubble in a Microchannel for High Heat Flux Cooling, J. Therm. Sci. Tech. 7 (2012) 740-752.

[19] L. Chen, X.R. Zhang, J. Okajima, S. Maruyama, Numerical Simulation of Stability and Heat Transfer Characteristics for Near-Critrical Fluid Microchannel Flows, Energ. Convers. Mgmt. 110 (2016) 407-418.

[20] L. Chen, X.R. Zhang, J. Okajima, S. Maruyama,Abnormal Microchannel Convective Fluid Flow near the Gas-Liquid Critical Point, Physica A 398 (2014) 10-24.

[21] L. Chen, X.R. Zhang, J. Okajima, S. Maruyama, Numerical Investigation of Near-Critical Fluid Convective Flow Mixing in Microchannels, Chem. Eng. Sci. 97 (2013) 67-80.

[22] T. Aihara, T. Ohara, T., and A. Sasago, Overall Performance of Heat Sink - Fan/Pump Unit and Its Optimum Design, Heat Trans. - Japanese Res. 26 (1997) 515-527.

[23] A.A. Alexeenko, D.A. Levin, S.F. Gimelshein, R.J. Collins, B.D. Reed, Numerical Modeling of Axisymmetric and Three-Dimensional Flows in Microelectromechanical Systems Nozzles, AIAA J. 40 (2002) 897-904.

[24] X. Hao, B. Peng, G. Xie, Y. Chen, Efficient on-chip hotspot removal combined solution of thermoelectric cooler and mini-channel heat sink, Appl. Therm. Eng. 100 (2016) 170-178.

[25] T. Muszynski, R. Andrzejczyk, Applicability of arrays of microjet heat transfer correlations to design compact heat exchangers, Appl. Therm. Eng. 100 (2016) 105-113.

[26] J. Gaviglio, Reynolds analogies and experimental study of heat transfer in the supersonic boundary layer, Int. J. Heat Mass Trans. 30 (1987) 911-926. 
[27] V. Lijo, H.D. Kim, and T. Setoguchi, Effects of Choking on Flow and Heat Transfer in Microchannels, Int. J. Heat Mass Trans. 55 (2012) 701-709.

[28] M.S. Shadloo, A. Hadjadj, F. Hussain, Statistical Behavior of Supersonic Turbulent Boundary Layers with Heat Transfer at Mœ=2, Int. J. Heat Fluid Flow 53 (2015) 113-134.

[29] Y. Takahashi, J. Okajima, A. Komiya, and S. Maruyama, Effect of Supersonic Micro Nozzle Shape on the Heat Transfer in a Surface Wall, Proc. 10th Int. Conf. Flow Dynamics, 2012, pp. 548-549.

[30] Y. Takahashi, J. Okajima, Y. Iga, A. Komiya, W.S. Fu, S. Maruyama, Study of Supersonic Microchannel for Cooling Electronic Devices, Proc. ASME 2013 11th Int. Conf. Nanochannels, Microchannels, Minichannels, 2013, Paper No. ICNMM 2013-73134.

[31] S. Maruyama, T. Shibata, K. Tsukamoto, Measurement of diffusion fields of solutions using real-time phase-shift interferometer and rapid heat-transfer control system, Exp. Therm. Fluid Sci. 19 (1999) 34-48.

[32] A. Komiya, and S. Maruyama, Precise and Short-Time Measurement Method of Mass Diffusion Coefficients, Exp. Therm. Fluid Sci. 30 (2006) 535-543.

[33] E. Shoji, A. Komiya, J. Okajima, H. Kawamura, S. Maruyama, High-Speed Phase-Shifting Interferometry Using Triangular Prism for Time-Resolved Temperature Measurement, Appl. Opt. 54 (2015) 6297-6304.

\section{Nomenclatures}

$\begin{aligned} A & =\text { area } \\ \mathrm{E} & =\text { energy } \\ f & =\text { friction factor } \\ h & =\text { height, enthalpy } \\ I & =\text { unit tensor } \\ k & =\text { thermal conductivity } \\ K & =\text { Gladstone-Dale constant } \\ l & =\text { optical length } \\ L & =\text { length }\end{aligned}$




$$
\begin{aligned}
n & =\text { refractive index } \\
N & =\text { fringe number } \\
p & =\text { pressure } \\
R & =\text { gas constant } \\
t & =\text { time } \\
T & =\text { temperature } \\
v & =\text { velocity } \\
X & =\text { x direction } \\
Y & =\text { y direction }
\end{aligned}
$$

Greek symbols

$$
\begin{array}{rll}
\lambda & = & \text { wave length } \\
\rho & = & \text { density } \\
\mu & = & \text { viscosity coefficient } \\
\lambda & = & \text { wavelength } \\
\tau & = & \text { stress tensor }
\end{array}
$$

Subscripts

$$
\begin{array}{ll}
0 & =\text { test/reference value, initial value } \\
\text { in } & =\text { inlet } \\
\text { out } & =\text { outlet } \\
t & =\text { time dependent value } \\
r & =\text { reference value } \\
\text { eff } & =\text { effective value }
\end{array}
$$




\section{Figure and Table Legends}

Fig. 1 Design and integration of microchannels. (a) straight channel; (b) bumped channel.

Fig. 2 Fabrication process of microchannels.

Fig. 3 Schematic diagram of the interferometric measurement and visualization system.

Fig. 4 Schematic diagram of the calculation domain for each microchannel designe. (a) Straight channel; (b) Bumped channel.

Fig. 5 Measurement results of the straight channel. (a) Phase-shifted data; (b) Density distributions.

Fig. 6 Measurement results of the bumped channel. (a) Phase-shifted data; (b) Density distributions.

Fig. 7 Comparison of the density distributions between the straight and the bumped channel.

Fig. 8 Flow and heat transfer parameter distributions in straight channel from numerical simulation. (a) Mach number; (b) temperature; (c) density.

Fig. 9 Flow and heat transfer parameter distributions in newly designed bump channel from numerical simulation. (a) Mach number; (b) temperature; (c) density.

Fig. 10 Predicted Temperature changes of the straight channel and newly proposed bump channel along the flow direction by numerical simulation.

Table 1 Estimated fringe number and number of data points from the Gladstone-Dale relation. 


\section{Figures}

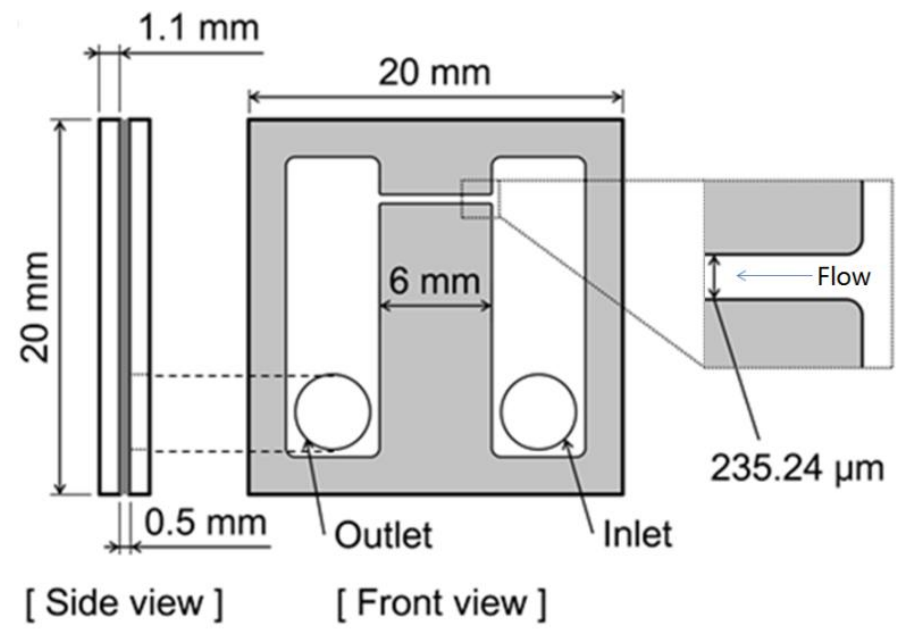

(a) straight channel

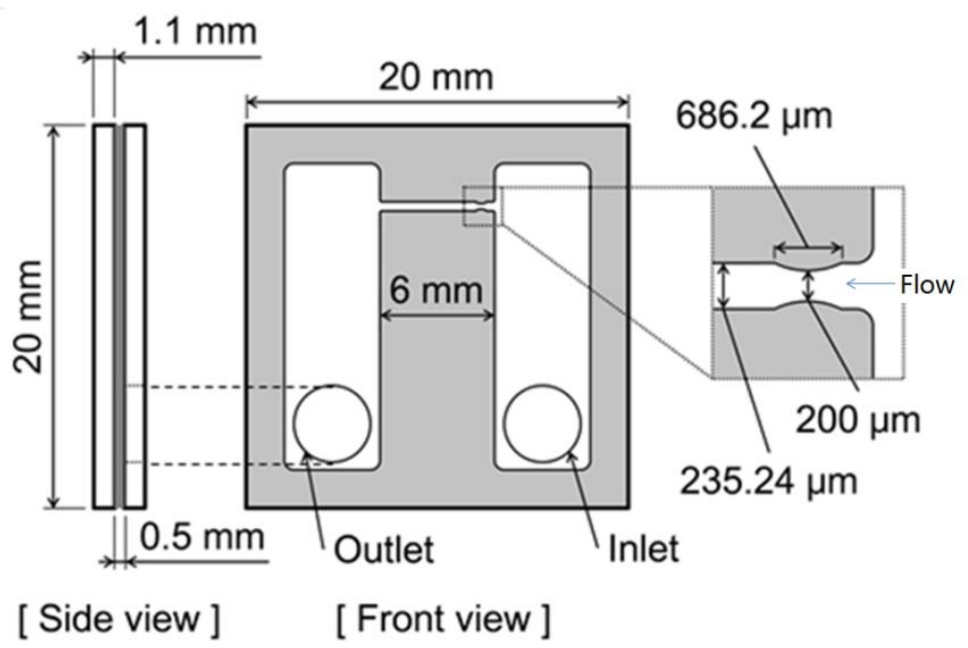

(b) bumped channel

Fig. 1 Design and integration of microchannels. (a) straight channel; (b) bumped channel. 
(a)

(b)

(c)

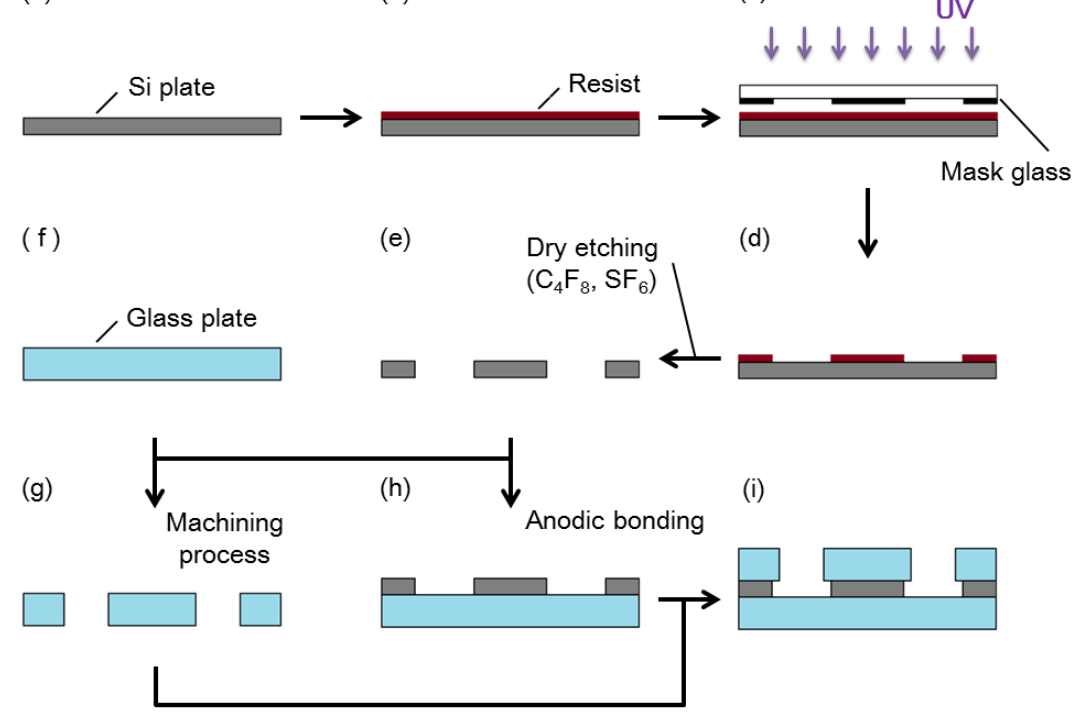

Fig. 2 Fabrication process of microchannels. 


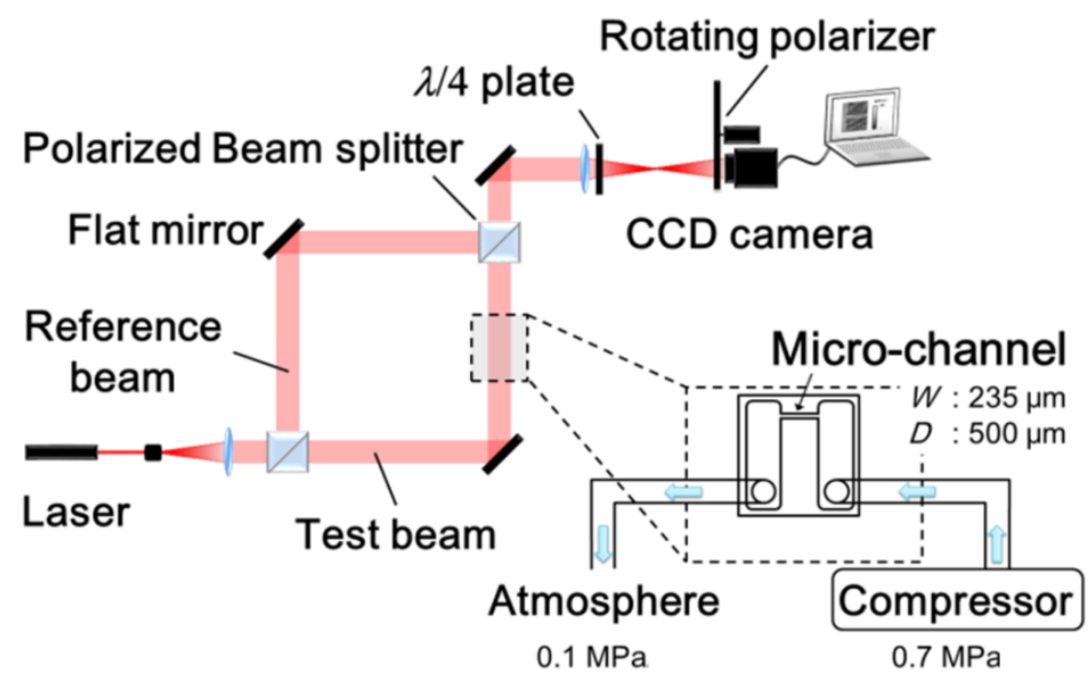

Fig. 3 Schematic diagram of the interferometric measurement and visualization system. 


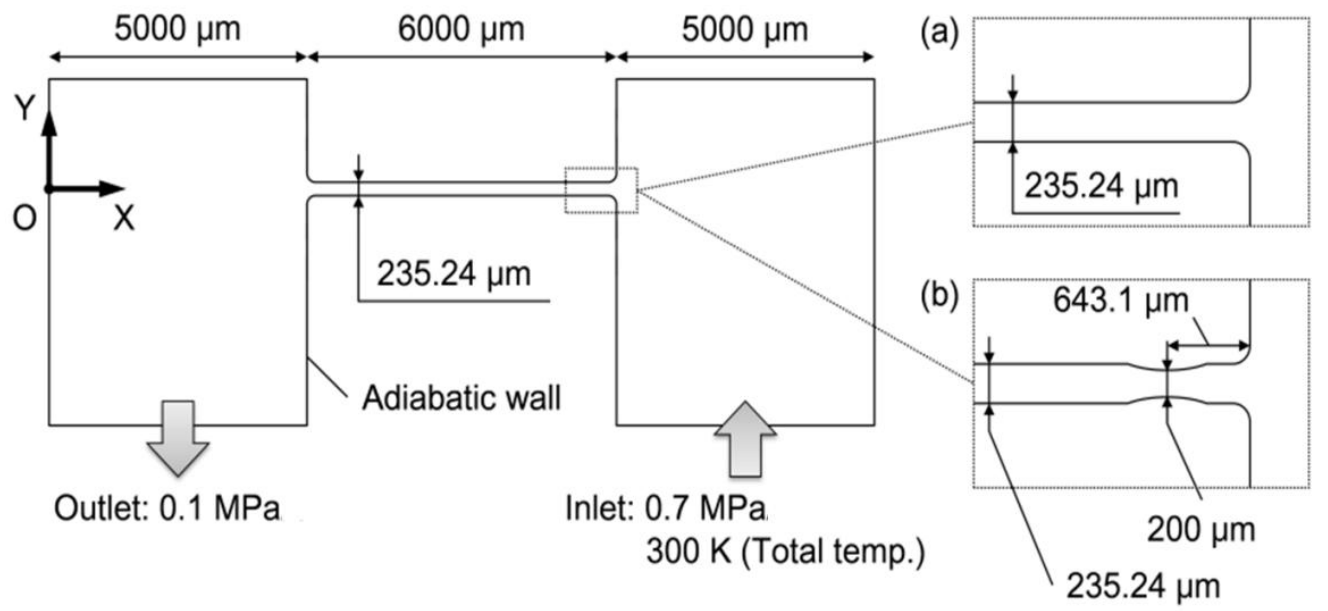

Fig. 4 Schematic diagram of the calculation domain for each microchannel designe. (a) Straight channel; (b) Bumped channel. 


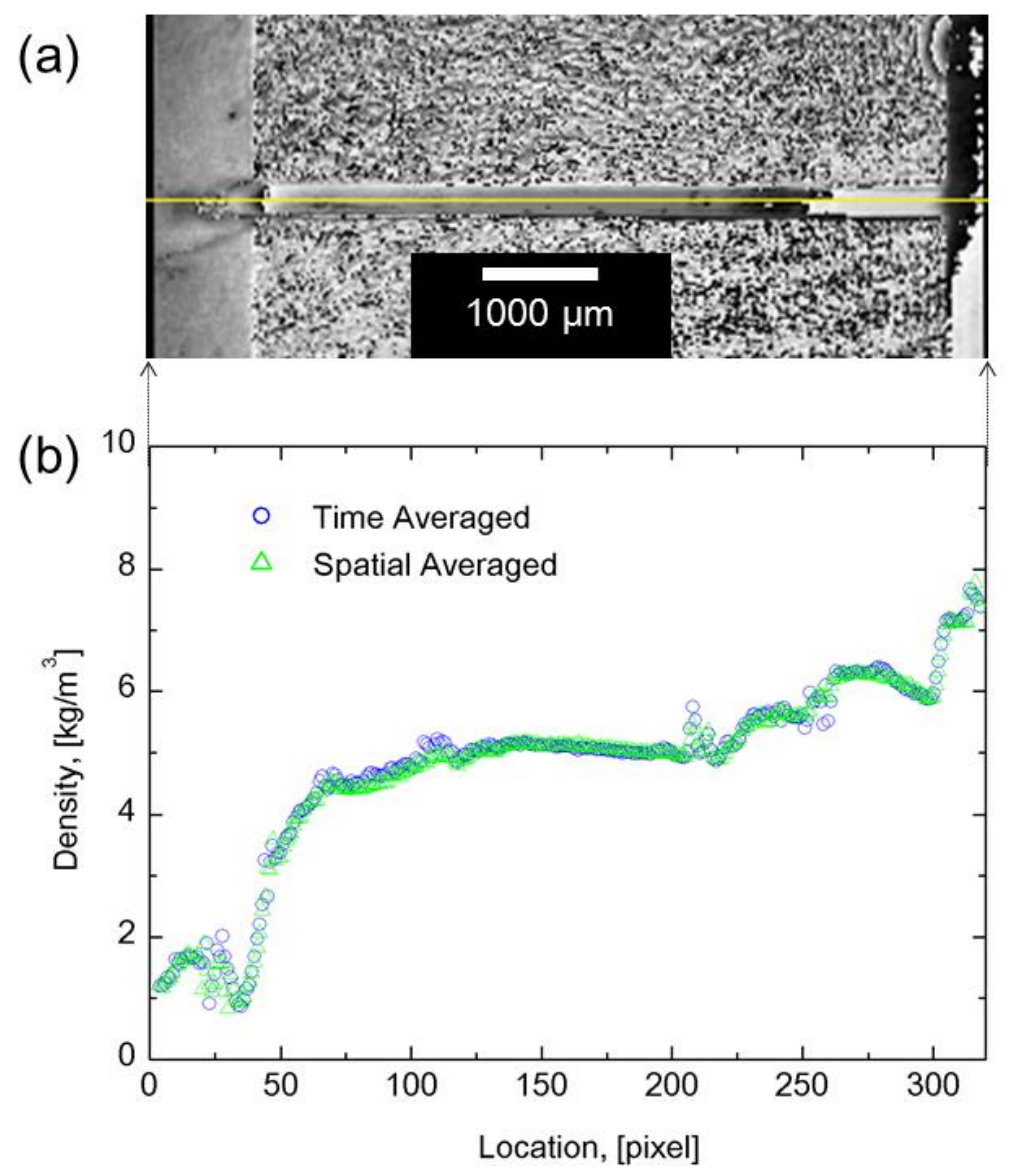

Fig. 5 Measurement results of the straight channel. (a) Phase-shifted data; (b) Density distributions. 


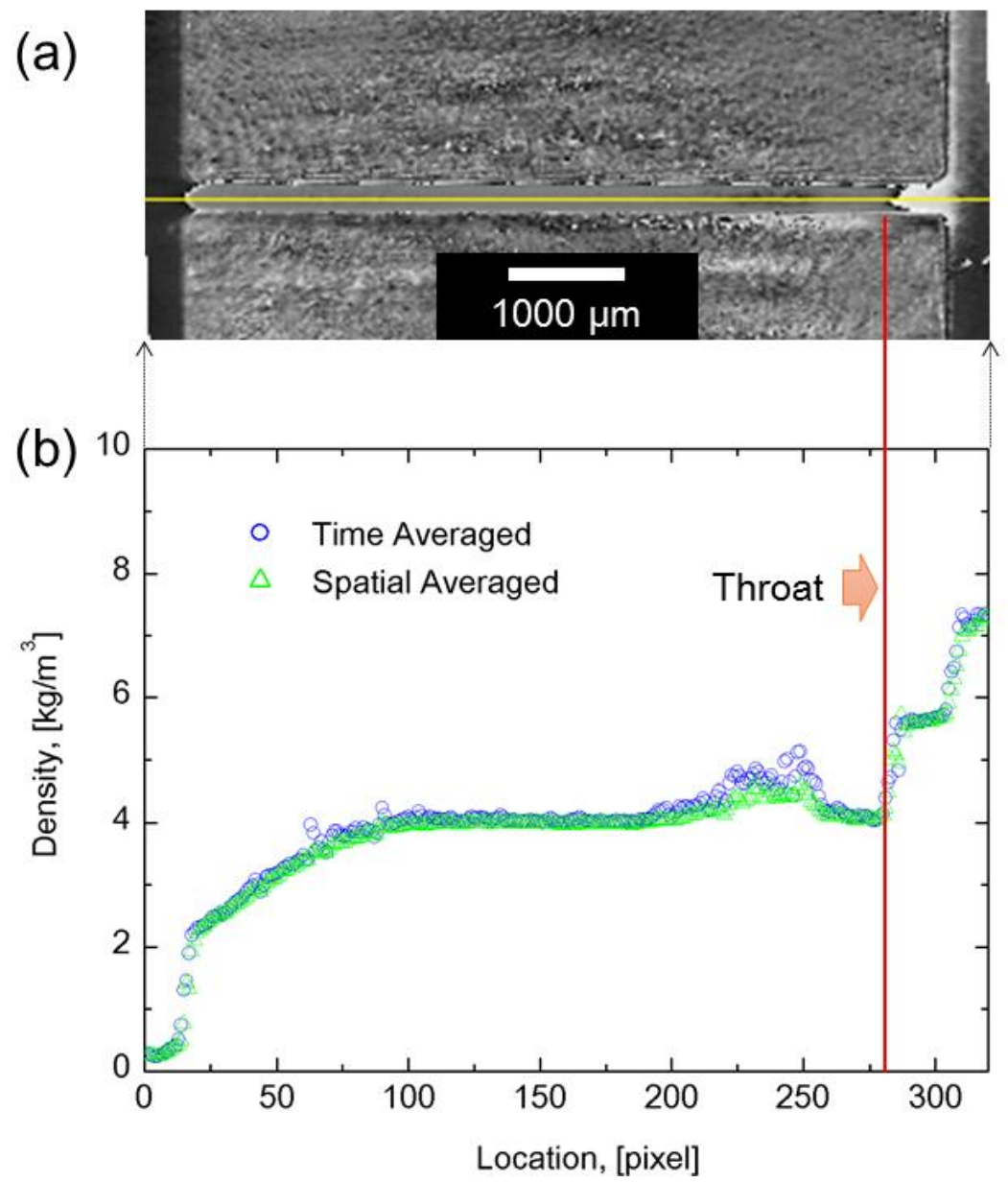

Fig. 6 Measurement results of the bumped channel. (a) Phase-shifted data; (b) Density distributions. 


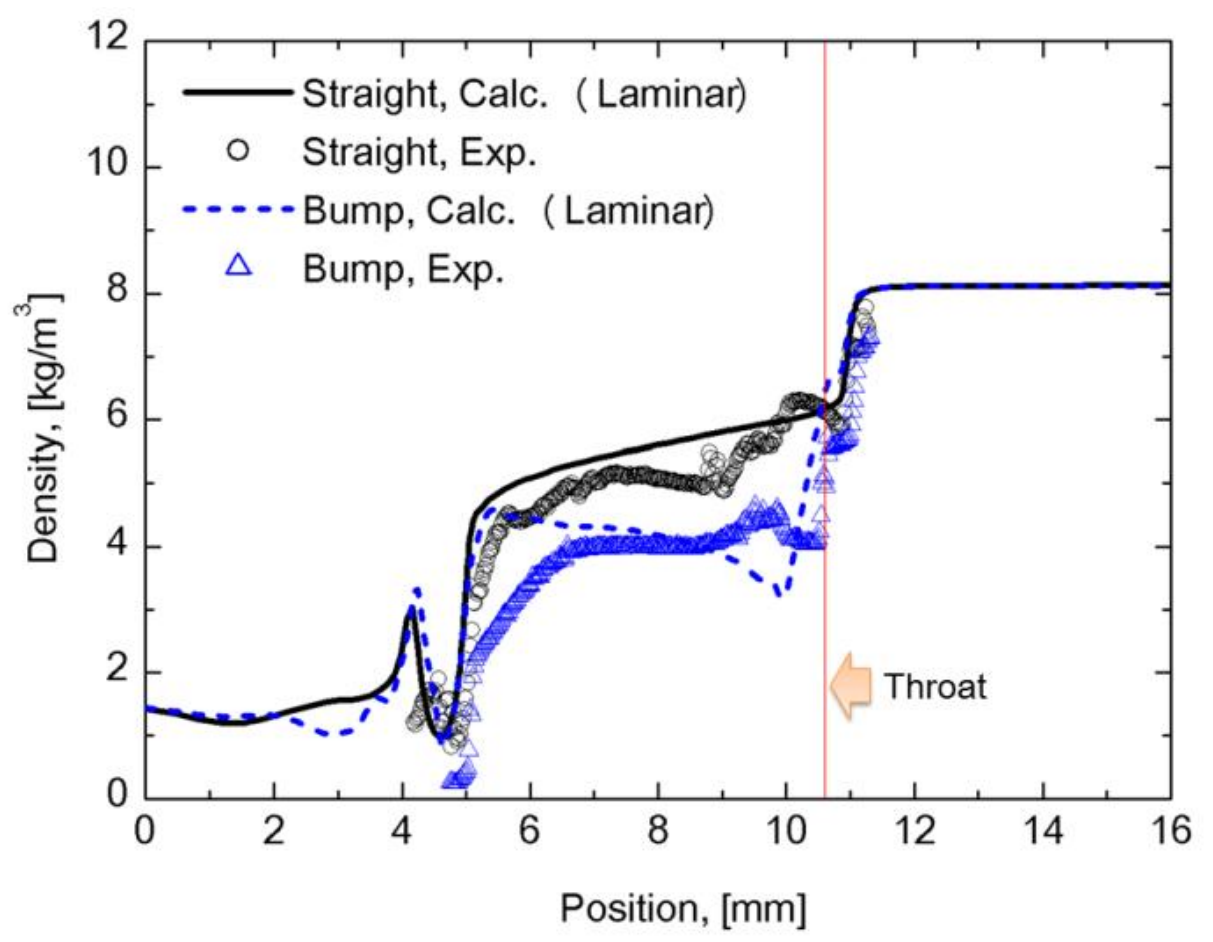

Fig. 7 Comparison of the density distributions between the straight and the bumped channel. 


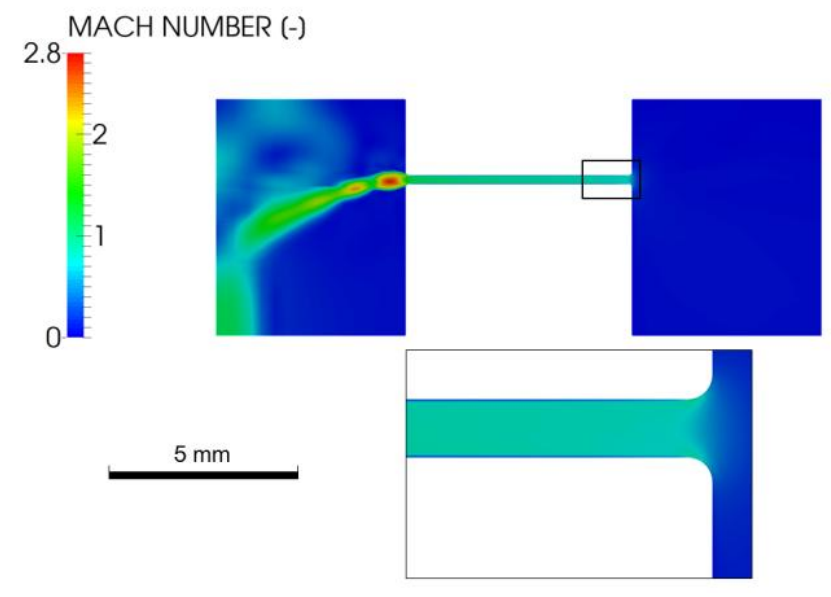

(a) Mach number

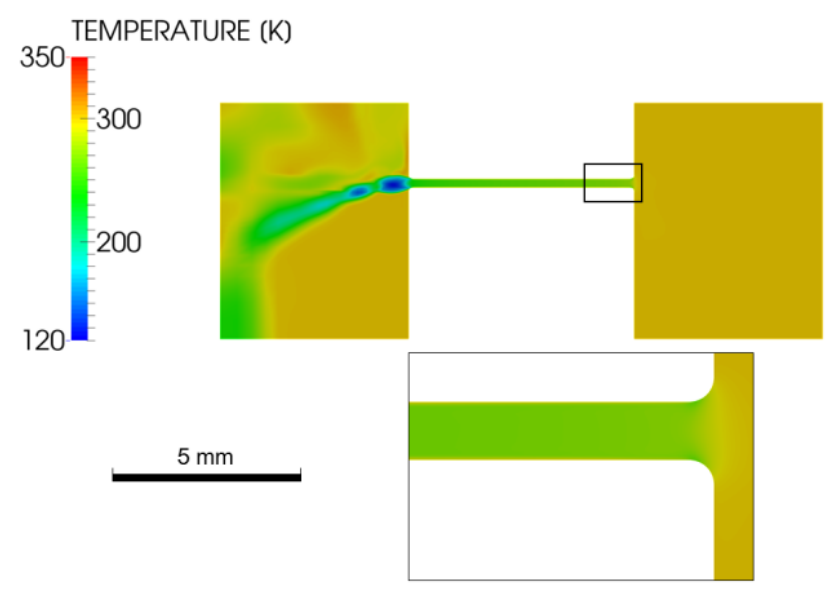

(b) temperature

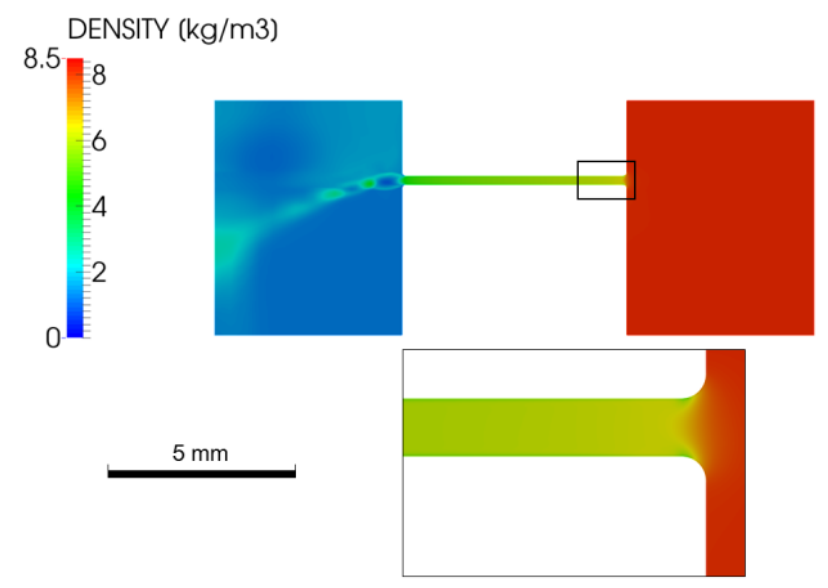

(c) density

Fig. 8 Flow and heat transfer parameter distributions in straight channel from numerical simulation. (a) Mach number; (b) temperature; (c) density. 


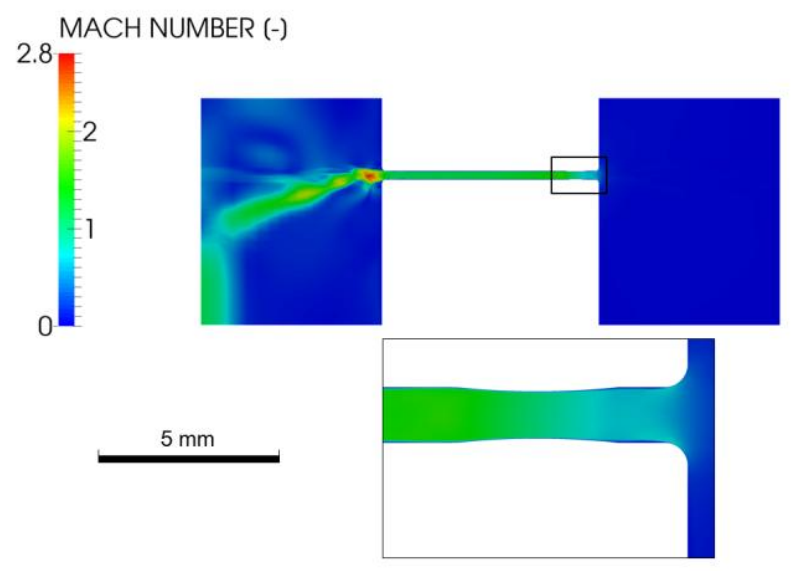

(a) Mach number

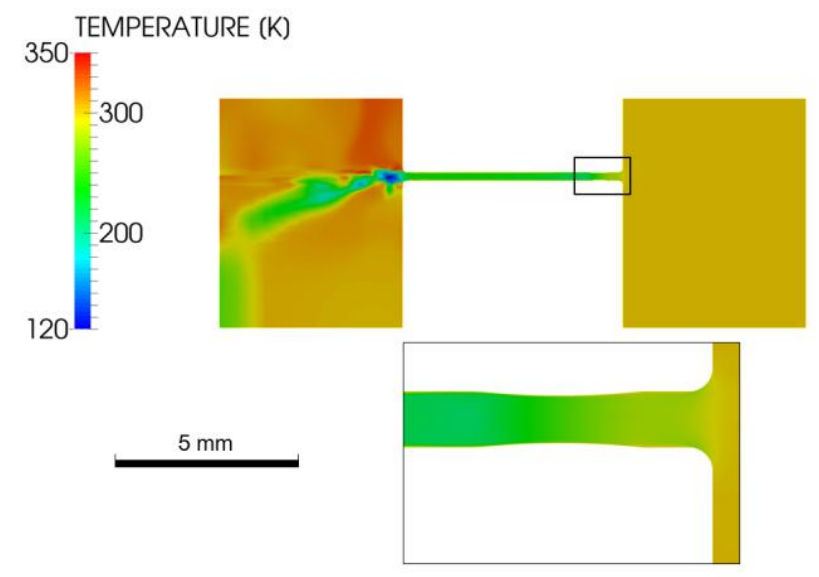

(b) temperature

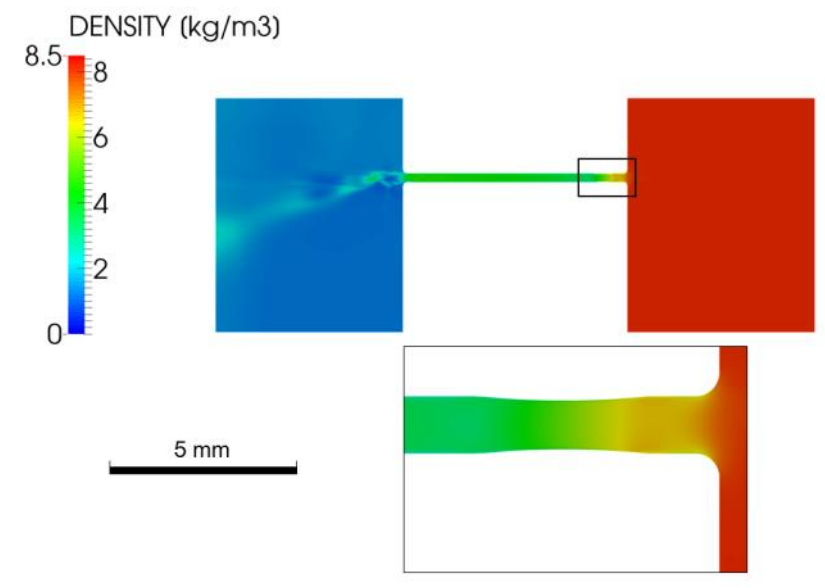

(c) density

Fig. 9 Flow and heat transfer parameter distributions in newly designed bump channel from numerical simulation. (a) Mach number; (b) temperature; (c) density. 


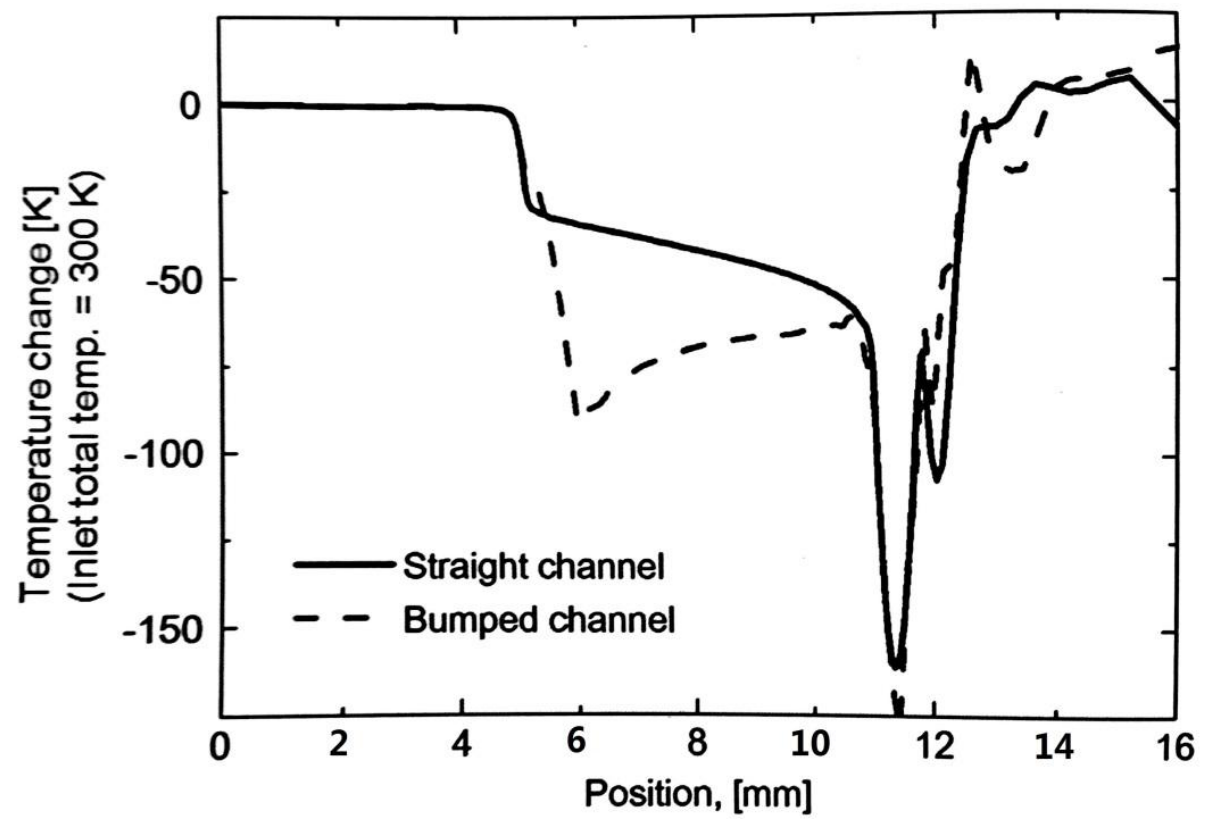

Fig. 10 Predicted Temperature changes of the straight channel and newly proposed bump channel along the flow direction by numerical simulation. 
Table 1 Estimated fringe number and number of data points from the Gladstone-Dale relation.

\begin{tabular}{|c|c|c|c|c|c|}
\hline \multirow{2}{*}{$\begin{array}{c}\text { Density } \\
{\left[\mathrm{kg} / \mathrm{m}^{3}\right]}\end{array}$} & $\begin{array}{c}\text { Refractive index } \\
{[-]}\end{array}$ & $\begin{array}{c}\text { Channel depth } \\
{[\mu \mathrm{m}]}\end{array}$ & $\begin{array}{c}\text { Fringe number } \\
{[-]}\end{array}$ & $\begin{array}{c}\text { Conventional } \\
\text { Interferometer } \\
{[\text { data number] }}\end{array}$ & $\begin{array}{c}\text { Phase-shifting } \\
\text { Interferometer } \\
{[\text { data number] }}\end{array}$ \\
\hline \multirow{2}{*}{1} & 1.000273 & - & - & - & - \\
\hline \multirow{3}{*}{2} & \multirow{3}{*}{1.000465} & 100 & 0.0605 & - & 15 \\
\cline { 3 - 6 } & & 500 & 0.302 & - & 77 \\
\cline { 3 - 6 } & \multirow{3}{*}{5} & 1000 & 0.605 & - & 310 \\
\hline \multirow{3}{*}{5} & 1.001161 & 100 & 0.281 & - & 72 \\
\cline { 3 - 6 } & & 500 & 1.40 & 1 & 359 \\
\cline { 3 - 6 } & & 1000 & 2.81 & 2 & 719 \\
\hline
\end{tabular}

